\title{
POLYGENIC FUNCTIONS OF HYPERCOMPLEX VARIABLES
}

BY P. W. KETCHUM AND TED MARTIN

1. Introduction. The purpose of this paper is to generalize to hypercomplex variables some of the results obtained by Kasner in the papers recently published by him on polygenic functions of the complex variable $z$ * Kasner has applied the term polygenic to any function of the form

$$
f(z)=\phi(x, y)+i \psi(x, y),
$$

where $\phi$ and $\psi$ are arbitrary functions (except for suitable continuity and differentiability assumptions) of the real variables $x, y$, the Cauchy-Riemann conditions not being assumed.

If the first derivative $d f / d z$, which is denoted by $\gamma=\alpha+i \beta$, be plotted in the $\alpha, \beta$ plane, the locus of the points $\gamma$ corresponding to a given point $z$ is a circle

$$
\begin{aligned}
& \left(\alpha-\frac{\phi_{x}+\psi_{y}}{2}\right)^{2}+\left(\beta-\frac{-\phi_{y}+\psi_{x}}{2}\right)^{2} \\
& =\left(\frac{\phi_{x}-\psi_{y}}{2}\right)^{2}+\left(\frac{\phi_{y}+\psi_{x}}{2}\right)^{2} .
\end{aligned}
$$

To the $\infty^{2}$ points of the $z$-plane correspond $\infty^{2}$ circles. This congruence of circles is called the Kasner derivative congruence. (In special cases where the function $f$ is monogenic the circles all shrink to points.)

Hedrick, Ingold, and Westfall $\dagger$ developed the theory of nonanalytic functions of a complex variable in a paper published in 1923 and E. R. Hedrick in a later paper $\ddagger$ pointed out relations

* E. Kasner, Theory of polygenic functions, Science, vol. 66 (1927), pp. 581582, and Proceedings of the National Academy of Sciences, vol. 14 (1928), pp. 75-82; Note on the derivative circular congruence of a polygenic function, this Bulletin, vol. 34 (1928), pp. 561-565.

$\dagger$ Hedrick, Ingold and Westfall, Theory of non-analytic functions of a complex variable, Journal de Mathématiques, (9), vol. 2 (1923), pp. 327-342.

$\ddagger$ E. R. Hedrick, On derivatives of non-analytic functions, Proceedings of the National Academy of Sciences, vol. 14 (1928), pp. 649-654. 
existing between the papers of Kasner and the paper of Ingold, Westfall, and himself.

Edna E. Kramer* has developed a theory analogous to this for polygenic functions of the dual variable $w=u+j v$,

$$
f(w)=\phi(u, v)+j \psi(u, v) .
$$

Mapping the derivative $d f / d w$ in the plane of $\gamma=\alpha+j \beta$, she obtained the congruence

$$
\beta=\frac{\phi_{v} \psi_{u}-\phi_{u} \psi_{v}}{\phi_{v}}+\frac{\psi_{v}+\phi_{u}}{\phi_{v}} \alpha-\frac{1}{\phi_{v}} \alpha^{2} .
$$

This equation represents a congruence of cycles (directed circles) in the coordinate system she used, but in the Cartesian coordinate system the congruence would be one of parabolas with axes parallel to the $\beta$-axis.

Analytic functions of hypercomplex variables have been studied by Scheffers $\uparrow$ for algebras which are commutative and associative. Ketchum $\ddagger$ has extended to analytic functions of hypercomplex variables all the important elementary theorems of complex variables. Antonne $\S$ has studied monogenic functions of variables in non-commutative algebras.

In $\S 2$ we shall give a generalization of the Kasner derivative congruence and in $\S 3$ we shall study some of the degeneracies of this generalized congruence.

2. The Kasner Derivative Congruence. We will denote the general hypercomplex variable by

$$
w=\sum_{i=1}^{n} x_{i} e_{i} ; \quad e_{i} e_{j}=\sum_{s=1}^{n} \gamma_{i j s} e_{s}, \quad(i, j=1, \cdots, n),
$$

where the units $e_{i}$ are linearly independent with respect to the complex domain, the constants $\gamma_{i j s}$ are real numbers, and the $x$ 's are complex variables.

* Edna E. Kramer, Polygenic functions of the dual variable $w=u+j v$, American Journal of Mathematics, vol. 52 (1930), pp. 370-376.

$\dagger$ Leipziger Berichte, vol. 45 (1893), p. 828.

$\ddagger \mathrm{P}$. W. Ketchum, Analytic functions of hypercomplex variables, Transactions of this Society, vol. 30 (1928), pp. 641-667.

$\S$ Journal de Mathématiques, (6), vol. 3 (1907). 
Consider the polygenic function

$$
f(w)=\sum_{i=1}^{n} e_{i} f_{i}\left(x_{1}, \cdots, x_{n}\right),
$$

where the $f_{i}$ are continuous functions of the variables $x_{1}, \cdots$, $x_{n}$ and possess continuous derivatives of the first order. We have the two derivatives

$$
d f=f^{\prime} d w, d f=d w f^{\prime},
$$

called the left-hand and right-hand derivatives respectively. If the algebra is commutative, the two derivatives are equal. We will, however, not assume that the algebra is commutative and we will develop the theory for the left-hand derivative. A parallel development could be carried out for the right-hand derivative, the first two subscripts on the $\gamma$ 's being interchanged throughout. From $\left(3_{1}\right)$ we obtain the relation

$$
\sum_{i=1}^{n} e_{i} d f_{i}=\sum_{k, j=1}^{n} e_{k} f_{k}^{\prime} e_{j} d x_{j}
$$

Hence

$$
\sum_{i, j=1}^{n} e_{i} \frac{\partial f_{i}}{\partial x_{j}} d x_{j}=\sum_{i, j, k=1}^{n} f_{k}^{\prime} e_{i} \gamma_{k j i} d x_{j} .
$$

Since the $e$ 's are linearly independent we can equate coefficients. We obtain $n$ linear homogeneous equations in the $n$ variables $d x_{j}$

$$
\sum_{j=1}^{n}\left(\frac{\partial f_{i}}{\partial x_{j}}-\sum_{k=1}^{n} f_{k}^{\prime} \gamma_{k j i}\right) d x_{j}=0, \quad(i=1, \cdots, n),
$$

which have a solution other than $0, \cdots, 0$ if and only if the determinant

$$
\left|\frac{\partial f_{i}}{\partial x_{j}}-\sum_{k=1}^{n} f_{k}^{\prime} \gamma_{k j i}\right|=0 .
$$

If (7) be considered as a function of the $n$ variables $f_{k}{ }^{\prime}$, it represents an $n$-parameter family of hypersurfaces, $M_{n}^{n}$. That is, for every point $x_{1}, \cdots, x_{n}$ there corresponds a hypersurface of $M_{n}^{n}$. Equation (7) is a generalization of the Kasner congruence 
for functions of ordinary complex variables. We propose to call this the Kasner derivative congruence for polygenic functions of hypercomplex variables.

For a given direction of differentiation, that is, for a given value of $d w$, unique values of $f^{\prime}$ will be determined by $\left(3_{1}\right)$, if and only if $d w$ is such a number in the algebra that left-hand division by it is possible. In other words $f^{\prime}$ will be determined uniquely if $d w$ is not a nilfactor. A necessary and sufficient condition that unique left-hand division by $d w$ is possible, as can be seen from (6), is that the determinant

$$
\Delta^{\prime}{ }_{d w}=\left|\sum_{j=1}^{n} \gamma_{k j i} d x_{j}\right| \neq 0 .
$$

If $\Delta_{d w}^{\prime}$ is zero, we must consider the rank of the two matrices

$$
M_{1}:\left\|\sum_{j=1}^{n} \gamma_{k j i} d x_{j}\right\| ; \quad M_{2}:\left\|\sum_{j=1}^{n} \gamma_{k j i} d x_{j}, \sum_{s=1}^{n} \frac{\partial f_{i}}{\partial x_{s}} d x_{s}\right\| .
$$

If the rank $r$ of the matrix $M_{1}$ is less than the rank of the augmented matrix certain restrictions are placed on the $d x_{i}$, that is, the derivative does not exist for certain paths of approach. If, however, $M_{1}$ and $M_{2}$ have the same rank $r$, which may happen at certain points or may be true for certain functions everywhere, then values of $n-r$ of the unknowns $f_{k}{ }^{\prime}$ may be assigned at pleasure and the others will be uniquely determined.

We will give an example of a function in an algebra of two units such that restrictions are placed on the direction of differentiation.

Let us consider the variable $w=i x+j y$ and the function $f(w)$ $=i \phi(x, y)+j \psi(x, y)$ in the algebra with the multiplication table

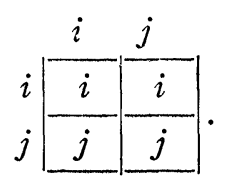

The matrix $\left\|\sum_{j} \gamma_{j k i} d x_{j}\right\|$ for this variable is

$$
\left\|\begin{array}{cc}
d x & d x \\
d y & d y
\end{array}\right\|
$$


which is of rank 1 . The corresponding augmented matrix for this variable is

$$
\left\|\begin{array}{lll}
d x & d x & \phi_{x} d x+\phi_{y} d y \\
d y & d y & \psi_{x} d x+\psi_{y} d y
\end{array}\right\|
$$

This matrix is of rank greater than 1 unless the relation

$$
\left|\begin{array}{ll}
d x & \phi_{x} d x+\phi_{y} d y \\
d y & \psi_{x} d x+\psi_{y} d y
\end{array}\right|=0
$$

holds, that is, unless

$$
m^{2} \phi_{y}+m\left(\phi_{x}-\psi_{y}\right)-\psi_{x}=0 .
$$

Thus we see that a restriction is imposed on the direction of differentiation.

This restriction also appears in the actual process of forming the right-hand derivative. We have

$$
\begin{aligned}
d f=d w f^{\prime}(w) & =(i d x+j d y)(i \alpha+j \beta), \\
i \phi_{x}+i \phi_{y} m+j \psi_{x}+j \psi_{y} m & =i \alpha+i \beta+j \alpha m+j \beta m .
\end{aligned}
$$

Equating coefficients, we obtain the two equations

$$
\phi_{x}+m \phi_{y}=\alpha+\beta, \psi_{x}+m \psi_{y}=m(\alpha+\beta),
$$

which show that the relation

$$
m^{2} \phi_{y}+m\left(\phi_{x}-\psi_{y}\right)-\psi_{x}=0
$$

must hold.

If we take the function $f(w)$ to be one such that $\phi_{y} \equiv \psi_{x} \equiv 0$ and $\phi_{x} \equiv \psi_{y}$, then the ranks of $M_{1}$ and $M_{2}$ are always the same, namely one, and the value of one of the two unknowns $\alpha$ or $\beta$ may be assigned at pleasure and the other will be uniquely determined. If at certain points the relations $\phi_{y}=\psi_{x}=0, \phi_{x}=\psi_{y}$ hold, then at those points the right-hand derivative will exist for every direction of approach and the value of one of the two unknowns may be assigned as in the previous case.

If we make the substitutions

$$
a_{i j}=\sum_{k=1}^{n} f_{k}^{\prime} \gamma_{k j i}, \quad b_{i j}=-\frac{\partial f_{i}}{\partial x_{j}},
$$


we can write the equation (7) as

$$
\begin{aligned}
\left|a_{i j}\right| & +\sum_{\substack{r=1 \\
j \neq r}}^{n}\left|a_{i j} b_{i r}\right|+\sum_{\substack{r, s=1 \\
j \neq r, s}}^{n}\left|a_{i j} b_{i r} b_{i s}\right|+\cdots \\
& +\sum_{\substack{r=1 \\
j \neq r}}^{n}\left|a_{i r} b_{i j}\right|+\left|b_{i j}\right|=0
\end{aligned}
$$

A consideration of equation (10) shows that the first term, $\left|a_{i j}\right|$, represents the terms of the $n$th degree in the variables $f_{k}{ }^{\prime}$, and since it depends only on the $\gamma$ 's, it is characteristic of the hypervariable. The determinant $\left|a_{i j}\right|$ is seen to be $\Delta_{f^{\prime}}$ where $\Delta_{f}^{\prime}$ is the right-hand determinant corresponding to (8) for righthand division. The equation

$$
\left|a_{i j}\right|=0
$$

determines the general nature of the hypersurfaces, since it determines the intersections of the hypersurfaces with the hyperplane at infinity. Thus we see that for a given algebra the hypersurfaces corresponding to any functions in the algebra are of the same general nature. The constant term, $\left|b_{i j}\right|$, involves only the derivatives of the function considered and is independent of the hypervariable. The intermediate terms depend on both the hypervariable and the function.

\section{Degeneracies of the Congruence.}

THEOREM. The hypersurfaces (7) will all go through the origin if and only if the jacobian of $f_{1}, \cdots, f_{n}$ vanishes.

From the equation of the congruence written in the form of equation (10) we see that the hypersurfaces all go through the origin when and only when the constant term is zero, that is, in view of the second relation of (9), when and only when the functions $f_{1}, \cdots, f_{n}$ are functionally dependent.

If $\left|b_{i j}\right|=0$, then for a fixed point in the plane of $x_{1}, \cdots, x_{n}$ the corresponding hypersurface in the plane of $f_{1}{ }^{\prime}, \cdots, f_{n}{ }^{\prime}$ will be tangent to the hyperplane

$$
\sum_{\substack{r=1 \\ j \neq r}}^{n}\left|a_{i r} b_{i j}\right|=0
$$

at the origin. 
If the rank of the jacobian matrix is $n-2$, that is, if there exist two relations (and not more) between the $f$ 's which do not involve the $x$ 's, the hypersurfaces will all have the origin as a double point, and terms of the second degree of (10) tell us the nature of the singular point.

We note that equation (7) would be satisfied if every element vanished. This would lead to $n^{2}$ linear equations in the quantities $f_{k}{ }^{\prime}$. It is known that if the algebra is commutative and associative, these $n^{2}$ equations have a unique solution for all analytic functions of $w$. The derivative congruence would in this case degenerate into a congruence of points, the function being monogenic.

UNIVERSITY OF ILLINOIS

\section{ON FINITE AND INFINITE COMPLETELY MONOTONIC SEQUENCES*}

\section{BY I. J. SCHOENBERG}

1. Introduction. As one of several important results concerning the problem of moments for a finite interval, Hausdorff $\dagger$ has proved the following theorem.

The problem of moments, which is to find a real function $\chi(t)$ for $0 \leqq t \leqq 1$, such that

$$
\mu_{n}=\int_{0}^{1} t^{n} d \chi(t), \quad(n=0,1,2, \cdots),
$$

in which the sequence $\mu_{0}, \mu_{1}, \mu_{2}, \cdots$ is given in advance, has a monotonic non-decreasing solution, if and only if

(2) $\Delta^{l} \mu_{m}=\sum_{\nu=0}^{l}(-1)^{\nu}\left(\begin{array}{l}l \\ \nu\end{array}\right) \mu_{m+\nu} \geqq 0, \quad$ for $l, m=0,1,2, \cdots$,

in which case the sequence is said to be completely monotonic.

The same problem (1) has a solution $\chi(t)$ which is of bounded variation, if and only if

* Presented to the Society, November 28, 1931.

$\dagger$ Ueber das Momentenproblem für ein endliches Interval, Mathematische Zeitschrift, vol. 16 (1923), pp. $220-248$. We refer to $\$ \S 1$ and 2 . 